Julia L. Carboni*, Saba Siddiki, Chris Koski

and Abdul-Akeem Sadiq

\title{
Using Network Analysis to Identify Key Actors in Collaborative Governance Processes
}

https://doi.org/10.1515/npf-2017-0012

\begin{abstract}
Collaborative governance is an increasingly popular form of governance. In theory, collaborative governance processes should be inclusive and value the perspectives of multiple stakeholders, including nonprofit organizations. In practice, this may not be the case. Recent work on representation in collaborative governance has found asymmetry in the way actor groups are descriptively and substantively represented in the collaborative governance process. We extend this line of work by employing network methods to create a diagnostic tool to identify which individual actors are substantively represented in collaborative governance processes over time. This tool is designed to systematically evaluate whether individual actors are under or overrepresented in collaborative governance. It provides a starting point for groups to discuss whether they are functionally inclusive and to understand whether non-inclusion is random or systematic. We apply the tool to collaborative governance in a regional food system and find variance in substantive representation by actor, indicating the collaborative governance process diverges from its inclusive design in practice. In particular, nonprofit organizations who are formally part of the collaborative governance process are not substantively represented in formal meetings to the same degree as city and county agencies.
\end{abstract}

Keywords: collaborative governance, stakeholder representation, substantive representation, diagnostic tool, affiliation networks

*Corresponding author: Julia L. Carboni, Maxwell School of Citizenship and Public Affairs, Syracuse University, Syracuse, New York 13244-1100, USA, E-mail: jlcarbon@syr.edu

Saba Siddiki, Maxwell School of Citizenship and Public Affairs, Syracuse University, Syracuse, New York 13244-1100, USA, E-mail: ssiddiki@syr.edu

Chris Koski, Department of Political Science and Environmental Studies, Reed College, Portland 97202-8199, USA, E-mail: ckoski@reed.edu

Abdul-Akeem Sadiq, School of Public Administration, University of Central Florida, Orlando 32816-2200, Florida, USA, E-mail: abdul-akeem.sadiq@ucf.edu 


\section{Introduction}

Are systems designed to be inclusive actually inclusive in practice? Representation is a crucial aspect of governance in democratic societies (Hero and Tolbert 1995). In collaborative governance, representation of diverse groups is theoretically incorporated into the design of structures and processes to encourage representation and participation of multiple stakeholder groups in the policy process (Emerson and Nabatchi 2015; Gazley, Chang, and Bingham 2010). This includes nonprofit organizations and other actors outside of government. For example, in the Department of Housing and Urban Development Continuum of Care programs, nonprofit providers work with state and local governments to secure housing for homeless individuals. In food policy councils, stakeholders- including nonprofits- come together to develop community level solutions to food system issues. The purpose of this article is to provide a diagnostic tool to assess whether collaborative governance processes are inclusive in practice.

While scholars have devoted considerable attention to the design of collaborative governance arrangements, it is not well understood whether or how various groups are substantively represented in practice (Koski et al. 2016). Diversity in representation is often framed as who has access to the collaborative process in terms of membership rosters. However, inviting actors to a collaborative process does not guarantee they will participate meaningfully in the process. While it is useful to understand who has a seat at the table, this does not give us much insight about who actually participates in collaborative governance arrangements.

Recent work investigating descriptive versus substantive representation by actor type found asymmetry in descriptive and substantive representation of actors by actor type (e. g. - nonprofit, government, business) (Koski et al. 2016). Specifically, not all actors who are formally part of collaborative governance arrangements participate in the process through things like meeting attendance or discussion participation. These findings indicate that while collaborative governance structures may be designed to include a diverse array of stakeholders as members, actual stakeholder representation may be much less diverse. This may have implications for collaborative outputs and outcomes. For example, if diverse groups are not substantively involved in the process, their interests and needs may be neglected in favor of the interests and needs of those who are substantively represented. This raises questions about whether there is systematic underrepresentation of specific groups or whether nonparticipation is more random. Systematic underrepresentation has implications for the governance process because collaborative governance arrangements are designed and legitimated as 
collaborative arrangements. Systematic underrepresentation undermines meaningful collaboration. Operationalizing and measuring who participates is fundamental to thinking about how to design and evaluate inclusivity in collaborative governance. Participation of individual actors should be measured relative to others in the collaborative governance process to get a sense of who is most central to collaborative governance arrangements.

The primary purpose of this manuscript is to provide a tool to systematically assess substantive representation of individual actors relative to their peers in collaborative governance arrangements over time. The tool will help academics and practitioners to understand if representation/non representation is systematic or random. Specifically, the tool provides a diagnostic to understand who is meaningfully represented in the process. Such diagnostics can serve as a starting point for understanding and discussing why some actors are represented or not represented. For instance, the result from the diagnostics may indicate that participation from a particular group of actors is repressed or that under representation may be the result of issues with individual actors who represent organizations. This tool provides a systematic analysis of participation in collaborative governance arrangements that allow collaborative groups to understand who has a voice at the table, instead of just who has a seat at the table.

In particular, we are interested in assessing whether interests of organizations outside of government - particularly nonprofit organizations- are represented in collaborative governance arrangements purportedly designed to include them. We focus on organizations rather than individuals because organizations are the members of collaborative governance arrangements. We extend previous work (Koski et al. 2016) by disaggregating actor types to individual organizational participants. We use network analysis methods to examine substantive representation. In the next section, we describe the setting for our application. Then we apply network methods to understand individual organizations' participation in collaborative governance arrangements over time.

\section{Setting and Case: Collaborative Policy Making in Food Systems}

Though collaborative governance pervades all levels of government and policy arenas, we study collaborative governance in local food systems. We chose food systems as our testbed because collaborative governance is becoming a dominant governance model in food systems. In addition, these systems are 
characterized by exceedingly complex issues, fraught with global, national, and local issues of growth, distribution, equity, and waste among others. Attempts to grapple with food system issues have largely occurred at the local level in the United States, where a diversity of stakeholders from a variety of sectors unite to address issues relating to agriculture, nutrition, social justice, commerce, and environmental protection among other things (Siddiki et al. 2015).

An emergent collaborative governance structure to contend with food system issues is the food policy council. Food policy councils are formal collaborative governance bodies that perform a variety of tasks related to the local food system. Though there is no standard council model, most bodies have representatives from different areas of the local food system and from multiple organizational sectors. Our particular case, which we refer to as the Western Food Policy Council (WFPC), has a mission to develop a just and integrated policy to enhance public health and regional food systems. In addition, the WFPC serves as a platform for engaging a diverse membership spanning the public, private, and nonprofit sectors. In this study, we use member and meeting data from the WFPC over one year.

The WFPC is an appropriate setting for developing a diagnostic tool to understand substantive participation in collaborative governance for several reasons. First, it consists of a diverse group of food system actors such as elected officials, tribal leaders, regional public health and economic development officials, food industry representatives, and a variety of public and nonprofit organizations (Johnston, Nicholas, and Parzen 2013). The explicit mission of the WFPC is to maintain a collaborative, holistic, and regional approach to addressing issues relevant to the food system. Second, the WFPC maintains a publicly accessible repository of council documents (e.g., monthly meeting minutes) amenable to coding for our analysis of representation in the council for illustrating the diagnostic value of the tool.

\subsection{Data Collection}

We used a mixed-method approach in collecting and analyzing data from council governance documents, meeting minutes, and individuals on the council. Our study period covered council meetings from (July 2013 to June 2014) and followed with interviews of key WFPC stakeholders in August 2014. To determine substantive representation in meetings, we collected data from WFPC meeting minutes and conducted hour-long semi-structured interviews with council participants. Publicly available meeting minutes were coded specifically to collect information on organizational attendance in meetings and discussion 
participation in meetings. The same individual (a WFPC staff member) took notes at each meeting. The organization met ten times during the year for about two hours in a centrally located facility. Because the organization spans multiple jurisdictions in a major metropolitan area, some members will occasionally call in. However, the vast majority of participation occurs in person at meetings that are open to the public. The meeting agendas are published in advance. Minutes record discussed topics, attendees (members and nonmembers), and participants in discussions.

Interviews were conducted with a purposive sample 13 members of the WFPC in August of 2014. In the interviews, we asked respondents to characterize a number of issues related to food policy councils broadly and the history of the WFPC. Specific to this research, we asked respondents questions regarding the influence of organizational structure on process and representation of different stakeholders in the WFPC. The interviews were designed to provide a reliability check on the use of minutes and to assist in helping to explain variation in representation and participation in council activities. We found interview responses to be consistent with the picture about representation that emerged in our diagnostic tool.

\subsection{Substantive Representation: Organizational Attendance and Participation}

Here we define substantive representation of organizations as attendance at meetings and participation in discussions at these meetings. ${ }^{1}$ In determining attendance, we looked at which council members (organizations) were present at each of the ten meetings. The focus of our work is the role of organizations as representative of interests; thus, we measure organizational attendance and participation rather than individual attendance and participation. This allows us to systematically analyze which organizations were substantively represented or not in meetings. From a diagnostic perspective, this enables us to isolate organizations who are under or over represented and begin to ask questions about representation that may lead to issues with the organization itself, individual performance, or suppression. This will lead to insights on how to balance participation more evenly.

As the food policy council membership is intentionally structured to represent particular interests and types of organizations, changes to individual members do not result in significant membership changes for the

1 This approach varies slightly from Koski et al. (2016). 
council. We consider an organization as attending a meeting if any representative of that organization (not a specific individual) attends a meeting. We coded meeting minutes to characterize organizational members' attendance and participation in meetings. Participation is coded as a substantive statement about an issue. We coded 139 such statements over 10 meetings. In this analysis, we do not examine topics discussed. For more information on topics, see Koski et al. (2016). There are 39 unique participants in meeting minutes.

\section{Using Network Methods to Understand Actor Centrality in Collaborative Policymaking}

To understand which actors are central to the collaborative process, we must examine the relative participation of actors. We use affiliation networks methods (Carboni 2015) to identify actors central to the collaborative governance process. Affiliation networks are multi-mode networks where there are two more distinct entities called modes (e.g. - actors, events). In the case of understanding substantive representation in collaborative processes, modes are members (organizations), meetings, and meeting participation. Affiliation network methods can be used to understand which organizations attend and participate in meetings over time and whether specific organizations are central to the collaborative process over time.

While we can observe these trends qualitatively, this method allows us to create an index and rank centrality of organizations relative to others engaged in the process to understand how organizations are represented and to determine whether under or over representation is systematic or random. Analyzing data in a more systematic way lends additional insights about simultaneous patterns of interactions among modes. In fully inclusive environments, organizations would have similar index scores that indicate relatively even participation among organizations over time; in less inclusive environments, there will be more variation in scores with underrepresented organizations having lower scores and over represented organizations having higher scores. In this section, we elaborate on how affiliation network matrices are created and analyzed.

Standard network analysis typically begins with a one-mode adjacency matrix where columns and rows are identical (Wasserman and Faust 1994). Cell entries $\left(\mathrm{x}_{\mathrm{ij}}\right)$ in the adjacency matrix reflect whether nodes are adjacent, or connected, with cell values of one or greater indicating a connection. In a two- 
mode affiliation matrix, rows and columns are not identical. Cell entries $\left(\mathrm{a}_{\mathrm{ij}}\right)$ reflect affiliations between actors in one mode (i. e. - organizations) with actors in another mode (i. e. - meetings). Typically, two mode matrices are reduced to one-mode adjacency matrices through matrix algebra for analysis because most data analysis techniques require a square matrix where rows and columns represent the same actors. The single mode adjacency matrix is square where the rows and columns represent the same entities- each node has a row and a column- and connections between nodes indicate common affiliation with a second mode (e. g.- an event). For example, in Breiger's (1974) seminal study on duality, southern women and parties were initially represented by a two mode matrix that was reduced to one mode to understand how parties created cliques among women. If one is to consider more than two simultaneously (e. g.organizations, meetings, meeting participation), a multi-mode matrix is necessary. Methods for multi-mode matrices are nascent, as most network literature has focused on single mode adjacency matrices or converting two-mode matrices (Borgatti and Everett 1997; Carboni, 2015). Reducing the matrix using three or more modes of data requires several extra steps to ensure adequate representation of the data.

Following Carboni (2015), we develop a valued two-mode matrix representing three modes of data- actors, meetings, and participation. Actors are represented in rows and meetings are represented in columns. Cell entries $\left(\mathrm{a}_{\mathrm{ij}}\right)$ values reflect the amount of substantive participation by an organization at each meeting. Accounting for multiple meetings simultaneously allows us to assess participation over time relative to other participants. Because collaborative governance is not typically a one-time meeting, assessing participation over time is crucial for understanding representation.

This matrix is represented graphically in Figure 1. Organizations are represented by nodes labeled with the "O" prefix. Meetings are represented by nodes labeled with the "M" prefix. Links between organization and meeting nodes indicate an organization attended and participated in a meeting. The size of organization nodes indicates the total number of substantive comments that organization made across all meetings. The thickness of lines between organization nodes and meeting nodes indicate how many comments the organization made at that specific meeting. From this graph, it is clear there is variance in individual organization participation in meetings over time. However, it is difficult to rank organizations based on their relative participation using the two-mode matrix and visual representation.

To understand which actors are key to the collaborative process, we transform the two-mode matrix by multiplying it by its inverse to create a one-mode adjacency matrix where organizations are represented in both rows and 


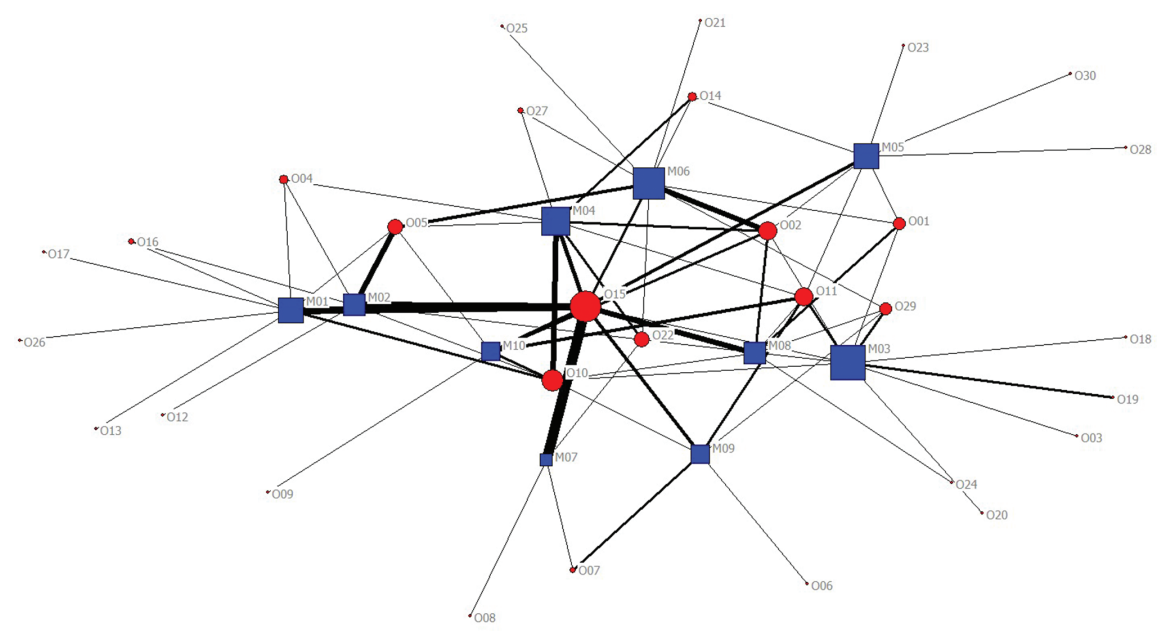

Figure 1: Valued two-mode affiliation network.

columns. Cross products are derived for each pair of organizations represented in the columns and rows by multiplying common participation in events between two actors and summing the result. The resulting adjacency matrix represents a single mode of organizations. The cell value $\left(\mathrm{x}_{\mathrm{ij}}\right)$ reflects the relative participation of each organization to others. A map of the matrix is given in Figure 2. Links between nodes indicate organizations participated in the same meetings. Thickness of lines between nodes indicates the number of meetings organizations participated in simultaneously. Node color represents the type of organization (e.g. - nonprofit, county government, university). Node shape represents the focus of the organization (e. g. - anti-hunger, extension, agriculture). Node size reflects centrality of organization in collaborative governance process over time. We discuss how this measure is derived below.

Following creation of the one-mode adjacency matrix, we create an index that reflects individual organization centrality within and across meetings relative to peers. We use weighted degree centrality which is the sum of weights for each node in the network (Barrat et al. 2004; Opsahl et al. 2008). This measure accounts for each organization's level of involvement within and across food policy council meetings. This index captures the breadth and depth of organization participation in meetings over time. The index is presented in Table 1. The index tells us which organizations are most central to this collaborative governance process. It is clear from the network map and index that the convener and city/county government organizations are most central to the process. We discuss these findings in the next section. 


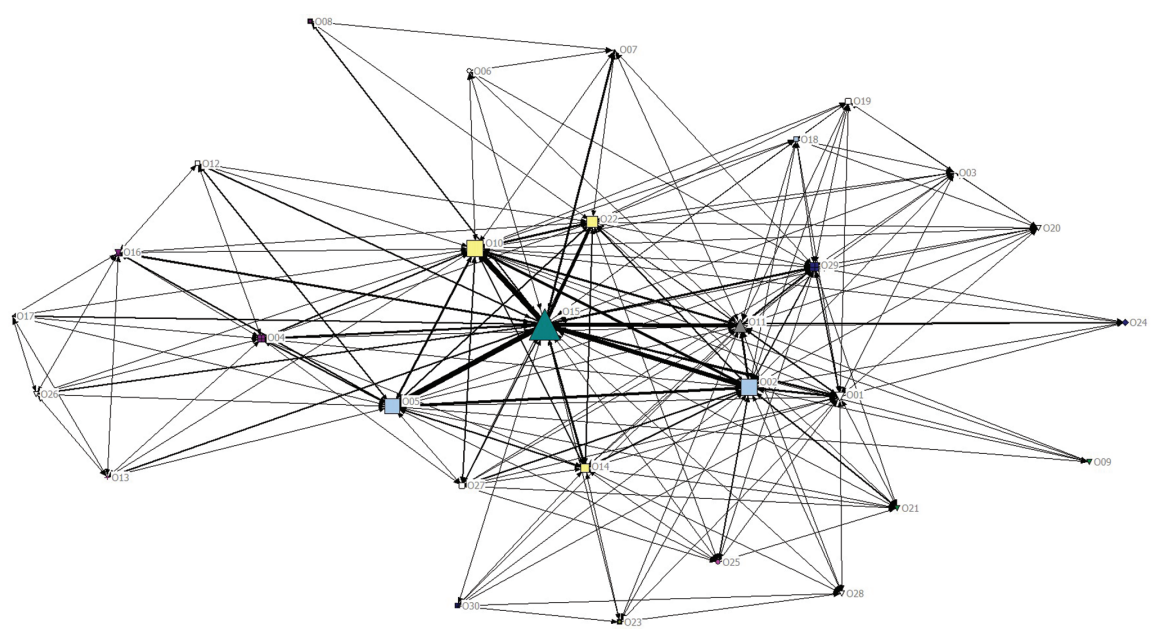

Figure 2: Valued one-mode affiliation network.

\section{Discussion}

After applying our diagnostic tool, we found that a group designed to be inclusive is not functionally inclusive. From the roster, it appears this group is representative of multiple stakeholder interests, but systematic analysis of organization participation within and across meetings tells a different story. The data tell us there is differential participation among stakeholder organizations within and across food policy council meetings. This variance is closely aligned with organizational sector; nonprofit organizations are relatively underrepresented while city and county governments are relatively overrepresented.

In arrangements where stakeholders are represented equally or nearly equally, scores would have little to no variance. However, in Table 1, we see large variance in centrality of organizations. The most active participants represent the convener and local government. It is notable that the most central node in the network is O15. This is the WFPC convener. Other central nodes are county governments (in yellow) and city governments (in light blue) while nongovernmental actors (nonprofits and business) operate at the periphery of the participation network. This is notable because it indicates that certain types of organizations - local and regional governments - participate more in the collaborative process than their private counterparts. Data demonstrate the process is driven by the convener and local governments. 
Table 1: Descriptive data and centrality scores.

\begin{tabular}{|c|c|c|c|}
\hline Organization & Organization TYPE & Organization focus & Centrality (normalized) \\
\hline 001 & Nonprofit & Resource conservation & 0.039 \\
\hline 002 & City government & Local department & 0.103 \\
\hline 003 & Nonprofit & Grocer & 0.008 \\
\hline 004 & Private & Food wholesale & 0.030 \\
\hline 005 & City government & Local department & 0.090 \\
\hline 006 & Nonprofit & Local food & 0.006 \\
\hline 007 & Nonprofit & Anti-hunger & 0.018 \\
\hline 008 & Private & Other & 0.007 \\
\hline 009 & Special district & Resource conservation & 0.007 \\
\hline 010 & County government & Local department & 0.106 \\
\hline 011 & Chair & Local legislature & 0.069 \\
\hline 012 & Nonprofit & Food access & 0.010 \\
\hline 013 & Private & Grocer & 0.008 \\
\hline 014 & County government & Local department & 0.038 \\
\hline 015 & Regional government & Regional department & 0.226 \\
\hline 016 & Private & Food production & 0.018 \\
\hline 017 & Nonprofit & Anti-hunger & 0.008 \\
\hline 018 & City government & Local department & 0.008 \\
\hline 019 & Nonprofit & Agriculture & 0.016 \\
\hline 020 & Nonprofit & Justice & 0.008 \\
\hline 021 & Special district & Resource conservation & 0.010 \\
\hline 022 & Country Government & Local Department & 0.058 \\
\hline 023 & Tribe & Tribe & 0.006 \\
\hline 024 & University & Higher education & 0.008 \\
\hline 025 & Other & Other & 0.010 \\
\hline 026 & Nonprofit & Resource conservation & 0.008 \\
\hline 027 & Nonprofit & Agriculture & 0.022 \\
\hline 028 & Nonprofit & Resource conservation & 0.006 \\
\hline 029 & University & Extension & 0.040 \\
\hline 030 & University & Extension & 0.006 \\
\hline
\end{tabular}


High descriptive representation paired with low substantive representation like what we found in our application of the diagnostic tool. This raises questions about the legitimacy and accountability of collaborative arrangements that are designed to be inclusive if the collaborative governance arrangement is legitimated on the premise of it being representative of multiple stakeholder groups.

\section{Conclusion}

We have developed a novel tool that practitioners and academics can use to assess the representativeness of organizations in collaborative governance processes. Our aim is to systematically analyze individual participation in groups so that groups can understand the locus of success or failure in representing the interests of stakeholders. Representation of interests is of crucial importance to any group, particularly groups that engage in collaborative processes, as an accountability mechanism. Collaborative processes by the nature of their existence appear to be inclusive to external constituents. However, representing multiple interests is challenging and actually showing which interests are represented even more so. Our tool is intended to help groups diagnose their representation in practice.

This tool would also be useful for understanding representation in other collaborative settings such as nonprofit boards where diversity is often prized but ends up being functional rather than inclusive (Brown 2002; BoardSource 2015; Freiwirth 2011). It should be noted that nonprofit board diversity is distinct from diversity in collaborative governance. In nonprofit boards, diversity is about individual participants (e. g. - race/ethnicity, gender). However this tool can be adapted to understand representation of diverse individuals in board meetings over time with modes as individual board members, some diversity variable, and participation over time. While there are many guides about promoting diversity and inclusivity, measuring actual representation of stakeholder groups is largely limited to self-reported data by executive directors and board chairs (BoardSource 2015). The tool developed in this paper can be used to systematically investiage whether boards are truly inclusive or only functionally inclusive, especially given requirements for nonprofit boards to keep meeting minutes.

In addition to understanding the level of inclusivity and substantive representation in specific groups, this tool can also contribute to building a larger understanding of representation in collaborative governance. It can be used to develop evidence in support of transparency. Groups may be monitored for 
inclusivity by those they purport to represent. This aligns with recent movements in public administration and policy such as the DATA Act (PL 113-101) for governance to be more open and transparent to actors not directly participating in the process.

This tool is not without limitations. Practitioners and groups members may have challenges in applying this tool to their current group given data constraints. While some groups with less complete recordkeeping may be challenged to use the tool to look back on previous representational dynamics, data collection for the tool we have presented here can be carried out going forward. Additionally, this tool only captures formal meeting participation. It does not capture interactions that take place outside of meetings. However, diagnosing whether groups are functionally inclusive may lead to discussions about more informal interactions or interactions outside meetings.

Groups may also choose to include information about individual participants. Another limitation of the case study presented here is our reliance on organizational representation rather than individual representation. Anyone who has participated in collaborative governance understands the power of personality in shaping group agenda. Moreover, individual characteristics such as race, class, and gender can influence intragroup dynamics. However, this diagnostic tool is designed to provide a starting point for discussion rather than a summative assessment of collaborative governance participation.

\section{References}

Barrat, A., M. Barthelemy, R. Pastor-Satorras, and A. Vespignani. 2004. "The Architecture of Complex Weighted Networks." Proceedings of the National Academy of Sciences 101 (11):3747-3752.

BoardSource. 2015. Leading with Intent: A National Index of Nonprofit Board Practices Series Number. Washington, D.C.: BoardSource.

Borgatti, S.P., and M.G. Everett. 1997. "Network Analysis of 2-Mode Data." Social Networks 19:243-269.

Brown, W.A. 2002. "Inclusive Governance Practices in Nonprofit Organizations and Implications for Practice." Nonprofit Management and Leadership 12:369-385. doi:doi:10.1002/ nml.12404.

Carboni, J.L. 2015. "Measuring Risks of Organizational Failure in Contract Exchange Structures.” Complexity, Governance and Networks 2 (1):45-64.

Digital Accountability and Transparency Act Of 2014. U.S. Public Law 113-101.

Emerson, K., and T. Nabatchi. 2015. Collaborative Governance Regimes. Washington, DC:

Georgetown University Press. 
Freiwirth, J. 2011. "Community-Engagement Governance: Systems-Wide Governance in Action.” Nonprofit Quarterly.

Gazley, B., W.K. Chang, and L.B. Bingham. 2010. "Board Diversity, Stakeholder Inclusion, and Collaborative Performance in Community Mediation Centers." Public Administration Review 70 (4):610-620.

Hero, R.E., and C.J. Tolbert. 1995. "Latinos and Substantive Representation in the U.S. House of Representatives: Direct, Indirect, or Nonexistent?" American Journal of Political Science 39:640-652.

Johnston, S., S. Nicholas, and J. Parzen. 2013. "Leading in the Community." In The Guide to Greening Cities, edited by J. Doe, 81-118. Washington, DC: Island Press/Center for Resource Economics.

Koski, C., S. Siddiki, A.-A. Sadiq, and J. Carboni. 2016. "Representation in Collaborative Governance: A Case Study of A Food Policy Council." American Review of Public Administration. doi:10.1177/0275074016678683.

Opsahl, T., V. Colizza, P. Panzarasa, and J.J. Ramasco. 2008. "Prominence and Control: The Weighted Rich-Club Effect.” Physical Review Letters 101:168702. doi:10.1103/ PhysRevLett.101.168702.

Siddiki, S., J.L. Carboni, C. Koski, and A.-A. Sadiq. 2015. "How Policy Rules Shape the Structure and Performance of Collaborative Governance Arrangements." Public Administration Review 75:536-547.

Wasserman, S., and K. Faust. 1994. Social Network Analysis: Methods and Applications (Structural Analysis in the Social Sciences). Cambridge University Press. 